\title{
Enriching the hierarchical model of achievement motivation: Autonomous and controlling reasons underlying achievement goals
}

\author{
Aikaterini Michou ${ }^{\prime *}$, Maarten Vansteenkiste ${ }^{2}$, \\ Athanasios Mouratidis ${ }^{3}$ and Willy Lens ${ }^{4,5}$ \\ 'Graduate School of Education, Bilkent University, Ankara, Turkey \\ ${ }^{2}$ Department of Psychology, Ghent University, Belgium \\ ${ }^{3}$ Department of Psychology, Hacettepe University, Ankara, Turkey \\ ${ }^{4}$ Department of Psychology, University of Leuven, Belgium \\ ${ }^{5}$ University of the Free State, Bloemfontein, South Africa
}

Background. The hierarchical model of achievement motivation presumes that achievement goals channel the achievement motives of need for achievement and fear of failure towards motivational outcomes. Yet, less is known whether autonomous and controlling reasons underlying the pursuit of achievement goals can serve as additional pathways between achievement motives and outcomes.

Aims. We tested whether mastery approach, performance approach, and performance avoidance goals and their underlying autonomous and controlling reasons would jointly explain the relation between achievement motives (i.e., fear of failure and need for achievement) and learning strategies (Study I). Additionally, we examined whether the autonomous and controlling reasons underlying learners' dominant achievement goal would account for the link between achievement motives and the educational outcomes of learning strategies and cheating (Study 2).

Sample. Six hundred and six Greek adolescent students $\left(M_{\text {age }}=15.05, S D=1.43\right)$ and 435 university students $\left(M_{\text {age }} M=20.5 \mathrm{I}, S D=2.80\right)$ participated in studies $\mathrm{I}$ and 2 , respectively.

Method. In both studies, a correlational design was used and the hypotheses were tested via path modelling.

Results. Autonomous and controlling reasons underlying the pursuit of achievement goals mediated, respectively, the relation of need for achievement and fear of failure to aspects of learning outcomes.

Conclusion. Autonomous and controlling reasons underlying achievement goals could further explain learners' functioning in achievement settings.

Some individuals may aim at outperforming their peers for challenge seeking, while others do so to validate their ego or to obtain a tangible reward. Such rationales can be conceived as proximal reasons for pursuing achievement goals as they are directly tied to the achievement goals themselves (Vansteenkiste, Lens, Elliot, Soenens \& Mouratidis, 2014).

*Correspondence should be addressed to Aikaterini Michou, Graduate School of Education, Bilkent University, 06800 Bilkent, Ankara, Turkey (email: aliki.michou@bilkent.edu.tr). 
From the perspective of Self-determination theory (SDT; Deci \& Ryan, 2000), one can autonomously pursue a goal (e.g., out of challenge or personal endorsement of the goal) or one can feel controlled by internally (e.g., avoiding feelings of guilt or shame) or externally (e.g., to be rewarded; to avoid punishment) pressuring reasons. Research (Gaudreau, 2012; Vansteenkiste, Mouratidis, \& Lens, 2010; Vansteenkiste, Smeets, et al., 2010) suggests that autonomous and controlling reasons underlying the pursuit of performance approach (PAp) or mastery approach (MAp) goals relate to different motivational correlates above and beyond the strength of approach goals themselves.

It remains relatively underexplored, however, whether similar relations apply for performance avoidance (PAv) goals and, more important, whether these relations fit within the hierarchical model of achievement motivation (Elliot, 1999). Herein, we suggest that the distal motives of need for achievement and fear of failure can, apart from instigating particular achievement goals, also activate proximal reasons undergirding the pursuit of achievement goals. Achievement goals and their underlying reasons are conceived to form a goal complex (Elliot, 2006) that represent two distinct pathways through which the need for achievement and fear of failure relate to learning outcomes. Specifically, we examined whether the achievement goals (i.e., the 'what' of goal pursuit) and their underlying autonomous and controlling reasons (i.e., the 'why' of goal pursuit; Vansteenkiste, Lens, et al., 2014) mediate the relation of need for achievement and fear of failure to learning correlates. Said differently, we attempted to incorporate the goal complex (i.e., the achievement goals and their underlying reasons) within the same integrated model to enrich the hierarchical model of achievement motivation (Elliot \& Church, 1997).

\section{Redefined achievement goals: Detaching reasons from aims}

Elliot (2005) conceived achievement goals as pure aims and distinguished them according to how competence is defined and valenced. When people define the required competence to attain success in terms of absolute or self-referenced standards of excellence and focus on approaching these standards, they endorse MAp goals. When they use the same criteria but focus on avoiding failure, they pursue mastery avoidance (MAv) goals. On the other hand, when people define competence with normative criteria (i.e., relative to the performance of others) and focus on attaining them, they endorse PAp goals, whereas when they use the same normative criteria but focus on avoiding failure they pursue PAv goals.

Elliot (2005) proposed a narrower goal definition by removing underlying reasons (e.g., 'proving one's ego'; 'getting a reward'; or 'seeking challenge') from the conceptualization of achievement goals. This delineated definition enables the examination of achievement goals separately from reasons undergirding their pursuit and thus lends conceptual clarity, uniform operationalization, and comparable results across different studies (Elliot, 2005; Hulleman, Schrager, Bodmann, \& Harackiewicz, 2010; Vansteenkiste, Lens, et al., 2014). Towards this end, Darnon, Dompnier, Delmas, Pulfrey, and Butera (2009; see also Urdan \& Mestas, 2006) showed that MAp, PAp, and PAv goals may be endorsed to maximize social gains or social utility. Following this suggestion (see also Husman \& Lens, 1999), we believe that jointly considering achievement goals as pure aims (what one strives for) and the underlying reasons (why one does so) is illuminating as it allows us examining whether achievement behaviour is primarily driven by the type of achievement goals one pursues, the reasons for pursuing them, or both. 
In line with Vansteenkiste, Lens, et al. (2014), we argue that the reasons underlying the pursuit of achievement goals can be understood in terms of the SDT (Deci \& Ryan, 2000), which distinguishes between autonomous and controlling reasons. Vansteenkiste, Smeets, et al. (2010) found that autonomous reasons for endorsing PAp goals - that is, pursuing PAp goals because they are interesting, challenging, or personally important - were associated positively to adaptive cognitive processing, metacognition, and engagement and negatively to anxiety and cheating. An opposite pattern was found for controlling reasons, that is pursuing PAp goals to attain contingent rewards, maintain self-worth, or avoid negative consequences. Interestingly, after controlling for their underlying reasons, PAp goals themselves yielded few unique associations with outcomes. Two other studies found autonomous reasons underlying PAp goals to be positively related to well-being (Gillet, Lafreniere, Vallerand, Huart, \& Fouquereau, 2012; Vansteenkiste, Mouratidis, et al., 2010) over and above the strength of PAp goal, while Gaudreau (2012) showed a similar pattern for MAp goals, as only MAp goals for relative autonomous reasons were linked with positive learning outcomes.

We aimed to build on this emerging body of research by enriching the hierarchical model of achievement motivation (Elliot, 1999). That is, we included the distal motives of need for achievement and fear of failure when investigating the associations among MAp, PAp, and PAv goals, their underlying autonomous and controlling reasons, and several learning correlates.

\section{The hierarchical model of achievement motivation}

The hierarchical model of achievement motivation (Elliot, 1999) posits that several constructs pertaining to competence, personal dispositions, and environmental factors influence, either independently or jointly, the adoption of a particular type of achievement goal. Herein, we focus on two competence-based variables, which occupy a central position in the achievement goal framework, that is individuals' dispositions to strive for success (i.e., need for achievement) and to avoid failure (i.e., fear of failure; Atkinson, 1957). In the hierarchical model, achievement goals represent the channels through which the acquired distal motives of need for achievement and fear of failure are manifested (Elliot, 1999).

Previous work indicates that the need for achievement, as an appetitive form of motivation, relates to MAp goals, whereas fear of failure, as an avoidance form of motivation, relates to PAv goals (Elliot \& Church, 1997; Elliot \& Murayama, 2008). Interestingly, both need for achievement and fear of failure have been found to relate positively to PAp goals as a means either to achieve success or to avoid failure, suggesting that PAp goals can be instigated by the two distal motives (Elliot \& Church, 1997). Given their energizing role, we expected need for achievement and fear of failure to relate not only to particular achievement goals but also to particular reasons underlying their pursuit.

\section{The present research}

Aligned with previous studies (e.g., Gaudreau, 2012; Gillet et al., 2012), we empirically separated achievement goals (i.e., the 'what' of achievement striving) from their underlying reasons (i.e., the 'why' of achievement striving). Apart from focusing on MAp 


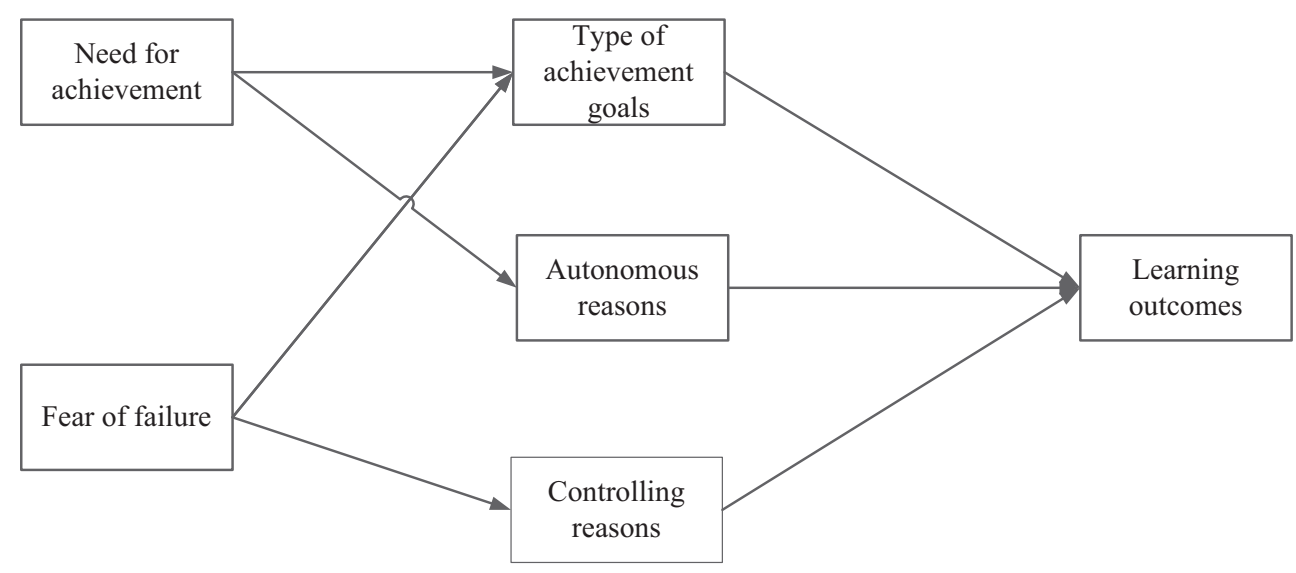

Figure I. The hypothesized enriched hierarchical model of achievement motivation.

and PAp goals, we also considered PAv goals and the reasons underlying their pursuit ${ }^{1}$ and we tested an enriched hierarchical model of achievement motivation (see Figure 1). Specifically, we investigated the relation of the distal achievement motives with both the goal content and the accompanied underlying reasons and their independent explanatory role in the relation of achievement motives to learning outcomes.

We hypothesized that need for achievement, as an approach tendency (Atkinson \& Feather, 1966), would relate positively to approach achievement goals. Additionally, we hypothesized that need for achievement would relate to autonomous reasons. This is because individuals high in the need for achievement are more likely to derive an intrinsic satisfaction from being successful (autonomous reason) in achievement tasks. Although individuals high in need for achievement may seek also contingent internal or external rewards (e.g., to boost their ego or to earn a prize, respectively), we believe that this is less likely for such individuals given the empirical evidence showing need for achievement to predict only the autonomous regulation of personally generated strivings (Sheldon \& Cooper, 2008).

We also expected fear of failure, as an inhibitory tendency (Atkinson \& Feather, 1966), to relate positively to PAv goals. Based on Elliot and Church (1997) who argued that striving for success could be a means to avoid failure (cf. Atkinson \& Feather, 1966; Lewin, 1938), we also expected fear of failure to relate positively to PAp goals. Additionally, we anticipated fear of failure to relate positively to controlling reasons because individuals high in fear of failure tend to act on the basis of pressuring incentives such as social disapproval, punishment, or anticipated feelings of shame and guilt (McGregor \& Elliot, 2005). In terms of SDT, these are controlling reasons for behaviour. We expected the relations between distal achievement motives and learning outcomes to be mediated by both the goal contents and their underlying reasons.

In Study 1, we tested the proposed enriched hierarchical model of achievement motivation (see Figure 1) where learners reported their autonomous and controlling reasons for MAp, PAp, and PAv goals and where the strength of endorsing achievement

\footnotetext{
I As the sample of Study I was middle and high school students, we avoided assessing MAv goals as we consider them to be more salient among elderly people (Ciani \& Sheldon, 2010).
} 
goals and their underlying reasons were modelled at the same level (i.e., as simultaneous mediators). We refrained from modelling them in a sequential order because of the correlational design of our study, ${ }^{2}$ although the 'what' and 'why' of achievement goals may influence each other over time, an issue that future longitudinal research needs to unravel. Also, we modelled them at the same level because we argue that the reasons are concomitantly undergirding the pursuit of achievement goals themselves, such that both the 'what' and the 'why' of achievement goals could play a role in the association between distal achievement motives and learning outcomes.

In Study 2, we no longer tapped into learners' goal strength but measured learners' goal preference via a dominant goal methodology (Van Yperen, 2006). Thus, in Study 2, learners' assessment of autonomous and controlling reasons was limited to their chosen dominant achievement goal. We chose to employ this method in the light of the strong inter-relationships among autonomous (and controlling) reasons underlying different achievement goals in Study 1. Also, by dividing learners based on their dominant goal choice, we could examine whether there would be substantial variation in the autonomous and controlling reasons within groups of learners preferring the same dominant goal, which could be predicted by the distal motives of need for achievement and fear of failure. Study 2 thus provided the opportunity to examine whether need for achievement and fear of failure would relate to autonomous and controlling reasons underlying learners' dominant achievement goal and whether these reasons would account for the relation between the distal achievement motives and learning strategies and cheating. As we aimed in Study 2 to examine the mediating role of reasons for each of the chosen dominant achievement goals separately, we did not model variability in chosen achievement goals as such and thus only tested a part of the model depicted in Figure 1 . Still, we deemed Study 2 as a useful addition that enabled us verifying the mediating role of the reasons underlying achievement goals in the hierarchical model of achievement motivation.

\section{STUDY I \\ Method}

\section{Participants and procedure}

Participants were 606 ( $45.4 \%$ males; five students omitted reporting their gender) middle and high school students $\left(M_{\text {age }}=15.05, S D=1.43\right)$ from five public schools located in an urban area in Greece. A permission to conduct the study was granted by the Greek Ministry of Education. The principals were contacted to get also their agreement, and an informed consent was obtained from parents. Students were assured that participation was confidential and voluntary. All students agreed to participate. The scales presented in a 5-point Likert type scale ( $1=$ Strongly disagree to 5 =Strongly agree $)$ were independently translated by two experts in the field and adjusted according to the procedures proposed by Hambleton (1994).

\footnotetext{
${ }^{2}$ We ran also two models in which goal contents and underlying reasons were modelled in a sequential way. In the first model, achievement goals are modelled as mediators in the relation between distal motives and underlying reasons. In the second model, achievement goals mediate the relation between underlying reasons and outcomes. The four-level models turn out to be fairly complex, and the core findings that we obtained in the three-level model are also obtained in these more complex models. Thus, we believe that the added value of building a four-level model is minimal as parsimony gets lost and the chance that a more complex model will be replicated in future work is lower.
} 


\section{Measures}

\section{Achievement motivation}

We used the short Achievement Motivation Scale (AMS; Lang \& Fries, 2006) to assess need for achievement (five items; e.g., 'I like situations in which I can find out how capable I am') and fear of failure (five items; e.g., 'I feel uneasy to do something if I am not sure of succeeding').

\section{Achievement goals}

We selected the revised Achievement Goal Questionnaire (Elliot \& Murayama, 2008) to assess pupils' MAp (three items; e.g., 'My aim is to completely master the material presented in this class'), PAp (three items; e.g., 'My aim is to perform well relative to other students'), and PAv goals (three items; e.g., 'My aim is to avoid doing worse than other students') in Mathematics or Modern Greek. We avoided assessing MAv goals given their low prevalence among adolescents (Ciani \& Sheldon, 2010).

\section{Underlying reasons of achievement goals}

Similar to Vansteenkiste, Mouratidis, et al. (2010), we asked students to indicate to what extent they pursued each of the given nine achievement goals for (1) intrinsic reasons (one item; e.g., 'because this goal is challenging and pleasant to me'); (2) identified reasons (one item; e.g., 'because I personally value this goal'); (3) introjected reasons (two items; e.g., 'because I can only be proud of myself if I do so';); (4) external reasons (two items; e.g., 'because others will reward me only if I achieve this goal'). Similar to previous research (Vansteenkiste, Mouratidis, et al., 2010; Vansteenkiste, Smeets, et al., 2010), we created an 'autonomous reasons' composite score for pursuing each of the three types of achievement goals by averaging the intrinsic and identified items. Likewise, we computed a 'controlling reasons' composite score by aggregating for each type of achievement goal the introjected and external items. Preliminary analyses revealed strong positive correlations among the autonomous reasons (range of $r=.65-.83$ ) and among the controlling reasons (range of $r=.82-.86$ ) underlying the three achievement goals. These high correlations imply that the way students regulate their MAp goals converges with the way they regulate their PAp and PAv goals, suggesting that their goal regulation is not necessarily coloured by the specific goal content. In the light of these findings, we collapsed the three scores for autonomous reasons underlying the pursuit of Map, PAp, and PAv goals into a single composite score and we did the same for the three scores for controlling reasons. Doing so yielded the advantage of avoiding problems of multicollinearity when the reasons underlying different achievement goals would be tested in a single path model.

\section{Motivated learning strategies}

We administered a part of the Motivated Strategies for Learning Questionnaire (MSLQ; Pintrich, Smith, Garcia, \& McKeachie, 1993) to assess three aspects of students' learning strategies: (1) critical thinking (five items; e.g., 'I often find myself questioning things I hear or read in this course to decide if I find them convincing'); (2) metacognitive self-regulation (five items; e.g., 'When I become confused about something I'm reading for my class, I go back and try to figure it out'); and (3) effort regulation (three items; e.g., 'I work hard to do well in this class even if I don't like what we are doing'). 


\section{Results}

\section{Preliminary analyses}

Descriptive statistics, bivariate correlations, and Cronbach alphas of the measured variables appear in Table 1. Confirmatory Factor Analysis of each scale showed acceptable fit. The results are available upon request. A MANOVA test showed significant gender differences, Wilk's $\Lambda=.891, F(10,558)=6.82, p<.01$, multivariate $\eta^{2}=.11$. A follow-up ANOVA with a Bonferroni alpha level adjustment showed significant gender differences in need for achievement $F(1,567)=12.25, p<.001, \eta^{2}=.02$, fear of failure $F$ $(1,567)=12.47, p<.001, \eta^{2}=.02$, and critical thinking $F(1,567)=16.65, p<.001$, $\eta^{2}=.03$. Females, as compared to males, scored higher in need for achievement $(M=4.06, S D=0.51$ vs. $M=3.88, S D=0.71)$ and fear of failure $(M=3.54, S D=0.71$ vs. $M=3.32, S D=0.77)$ and lower in critical thinking $(M=2.97, S D=0.76$ vs. $M=3.24, S D=0.80$ ). Given these findings, gender was included as a covariate in the subsequent analyses.

\section{Main analyses}

We tested the hypothesized theoretical model in Figure 1 through path analysis with EQS 6.1 software, Multivariate Software, Inc., Encino, CA, USA. The model showed acceptable fit $\left(\mathrm{S}-\mathrm{B} \chi^{2}[18, N=569]=57.95, p<.01, \quad \mathrm{CFI}=.971, \quad\right.$ SRMR $=.045$, RMSEA $=.063$ [90\% CI: .045-.081]). However, allowing direct paths from need for achievement to metacognitive self-regulation and to critical thinking improved the fit of the model $\left(\mathrm{S}-\mathrm{B} \chi^{2}[17, \quad N=569]=31.33, \quad p<.01, \quad \mathrm{CFI}=.989, \quad\right.$ SRMR $=.031$, RMSEA $=.039$ [90\% CI: .016-.059]). Given that previous studies have reported such direct paths from need for achievement to learning strategies (Michou, Mouratidis, Lens, \& Vansteenkiste, 2013), we accepted the improved model (see Figure 2). As hypothesized, need for achievement was positively related to approach goals and to autonomous reasons underlying the three achievement goals. In turn, MAp goals associated positively with effort regulation and metacognitive self-regulation. As expected, autonomous reasons were associated positively with all the three learning strategies. In contrast, fear of failure was positively correlated with PAv and PAp goals as well as with controlling reasons for pursuing achievement goals. Contrary to our hypothesis, no path from PAp, PAv goals, and controlling reasons to learning strategies was significant. A test of indirect effects showed that need for achievement was indirectly associated with effort regulation $(\beta=.15, z=6.91, p<.01)$, and metacognitive self-regulation $(\beta=.15, z=6.73, p<.01)$ by means of both MAp goals and autonomous reasons and with critical thinking $(\beta=.09, z=4.80, p<.01)$ by means of autonomous reasons only. ${ }^{3}$

\footnotetext{
${ }^{3}$ In a set of supplementary analyses, we examined to what extent autonomous and controlling reasons moderate the relation between achievement goals and the studied correlates. These moderated regression analyses revealed one significant interaction between MAp goals and autonomous reasons in the prediction of effort regulation, $\mathrm{B}=0.15,(\beta=10) \mathrm{SE}=0.06, \mathrm{t}=2.40$, $\mathrm{P}<.05$. A test of simple slopes indicated that after controlling for gender, need for achievement and fear of failure, MAp goals were positively associated with effort regulation when autonomous reasons were high $(\mathrm{B}=0.38, \mathrm{SE}=0.06$, t-test of simple slopes $\mathrm{t}=6.32, \mathrm{p}<.0 \mathrm{I})$ or moderate $(\mathrm{B}=0.25, \mathrm{SE}=0.07$, t-test of simple slopes $\mathrm{t}=3.88, \mathrm{p}<.0 \mathrm{I})$ but not low $(\mathrm{B}=0.12, \mathrm{SE}=0.10$, t-test of simple slopes $\mathrm{t}=1.25, \mathrm{p}=.21, \mathrm{~ns})$. This interaction was in line with Gaudreau's $(2012)$ and Benita, Roth, and Deci (20I4) recent finding and suggests that MAp goals may be particular useful - at least for some outcomes when they are pursued for autonomous reasons.
} 


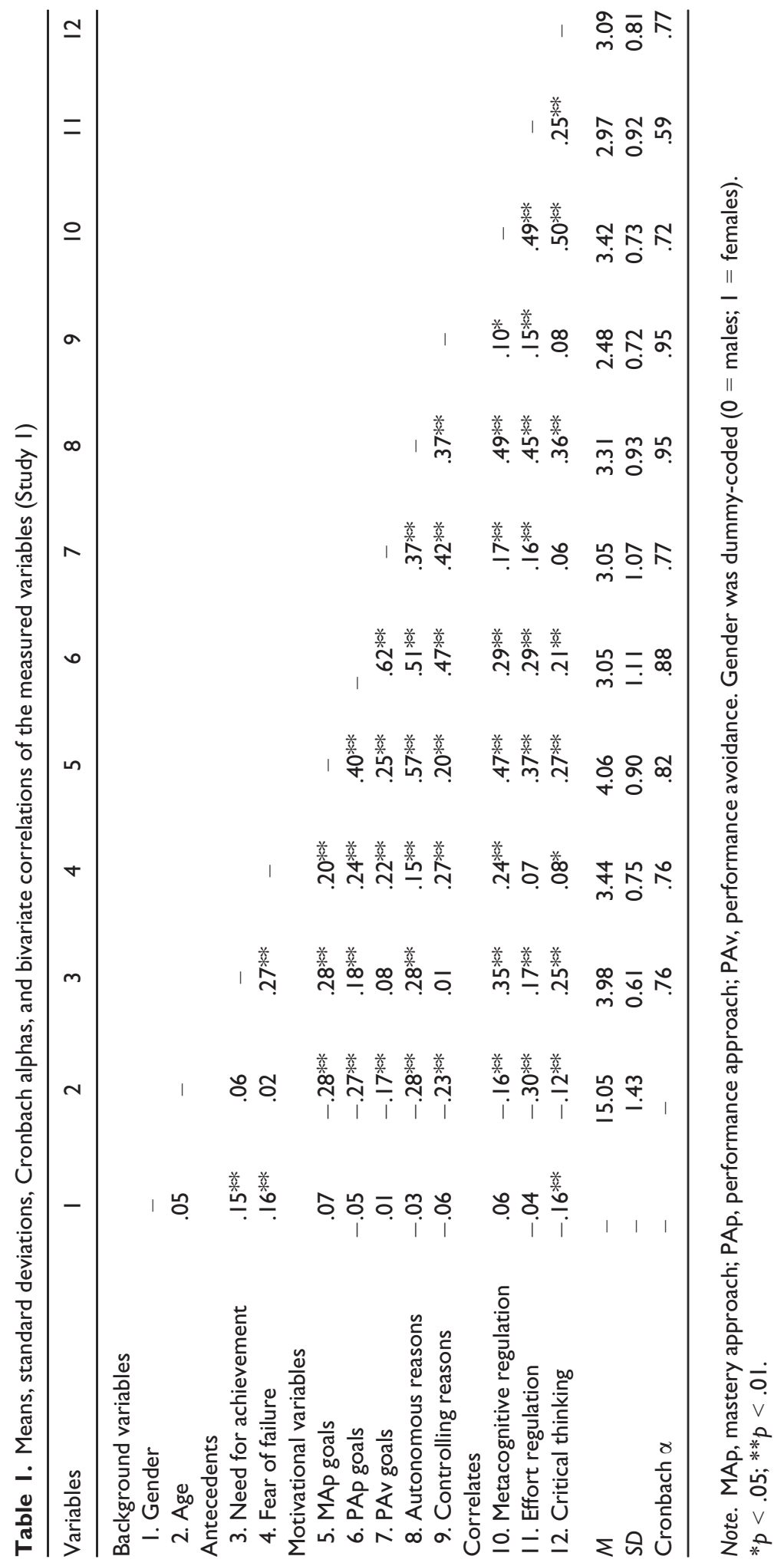




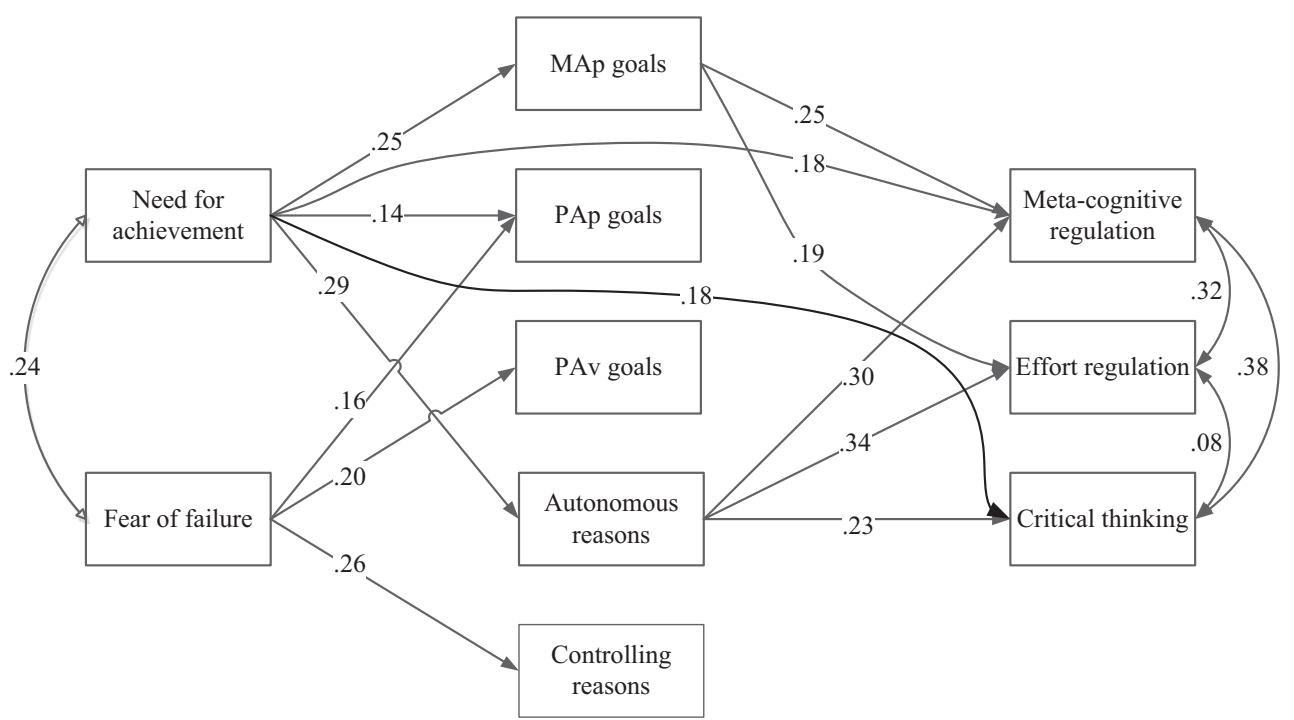

Figure 2. The Tested Model of Study I controlling for gender differences (not shown for sake of clarity). Also, not shown for sake of clarity are the correlations between MAp and PAP $(\beta=.35)$ as well as PAv goals $(\beta=.23)$; between MAp goals and autonomous $(\beta=.55)$; PAp goals to PAv goals $(\beta=.61)$; PAP goals to autonomous $(\beta=.49)$ and controlling reasons $(\beta=.44)$; PAv goals to autonomous $(\beta=.36)$ and controlling reasons $(\beta=.40)$ and autonomous to controlling reasons $(\beta=.38)$. All paths are standardized and significant at the .05 level.

\section{Brief discussion}

In line with our hypotheses, need for achievement related positively to both MAp and PAp goals and to autonomous reasons underlying achievement goals. Fear of failure related to PAv as well as to PAp goals and controlling reasons underlying the pursuit of achievement goals. Moreover, MAp goals and autonomous reasons played a mediating role between need for achievement and learning strategies, underscoring the explanatory power of both the 'what' and 'why' of achievement goals. Contrary to our expectations, PAp and PAv goals did not play any mediating role between dispositional motives and learning strategies. This finding is partially in accord with Vansteenkiste, Smeets, et al. (2010) who found that PAp goals did not account for any independent variance in educational outcomes when MAp goals and reasons underlying PAp goals were considered. Regarding controlling reasons, as they were unrelated to effective learning strategies, it will be illuminating to investigate whether they would perhaps relate positively to maladaptive educational outcomes like cheating, an issue we explored in Study 2.

Study 1 denotes that need for achievement and fear of failure may predict, respectively, autonomous and controlling reasons in a uniform fashion and irrespective of the goal to which these reasons are tied. This could be the reason of the high correlations between corresponding reasons underlying different types of achievement goals. However, to ensure that the obtained patterns of relations would occur also while learners have their primary achievement goal in mind, we asked participants in Study 2 to choose their dominant achievement goal and to report their autonomous and controlling reasons only for this dominant achievement goal. 


\section{STUDY 2}

We considered it important to test again the mediating role of autonomous and controlling reasons underlying the separate achievement goals. The dominant goal method (Van Yperen, 2006) allowed us to examine whether the variation in the underlying reasons of the same type of achievement goal would predict variation in learning outcomes, while being itself predicted by the distal motives. Unlike Study 1, we surveyed university students because we believed that students of that age can better critically reflect about their most dominant goal in academics and the reasons for which they endorse it. We also included cheating to keep some balance between positive (i.e., metacognition, effort regulation, and critical thinking) and negative indices of learning behaviour. This will permit us to check further the mediating role of controlling reasons between fear of failure and educational correlates.

\section{Method}

\section{Participants and procedure}

Thirty-five male and 400 female university students (along with five who omitted reporting their gender) participated in Study 2 . The students ( $M_{\mathrm{age}}=20.51$ years, $S D=2.80$ ) were coming from the Faculty of Education of a large Greek University. Participants completed the questionnaires during a regular class hour. A research assistant explained the scope of the study and stressed that participation was voluntarily and confidential. No student denied participation. Except the scale assessing the dominant achievement goal, all the questions were presented in a 5-point Likert type scale $(1$ = Strongly disagree to 5 = Strongly agree $)$.

\section{Measures}

\section{Achievement motivation}

Similar to Study 1, we used the 10-item AMS scale (Lang \& Fries, 2006) to assess students' need for achievement and fear of failure.

\section{Dominant achievement goal}

To assess students' most salient achievement goal, we used a method similar to that of Van Yperen (2006). Specifically, we selected from the AGQ-R (Elliot \& Murayama, 2008) one Map goal ('My aim is to completely master the material presented in this class'), one Pap goal ('My aim is to perform well relative to other students'), and one PAv goal ('My aim is to avoid doing worse relative to other students') and asked students to indicate which of the three goals they most strongly endorsed in their studies. Because assessing the dominant achievement goal through a 3-item scale may cast some doubt about the reliability of our assessment, we presented for a second time, and on a separate page, this set of three items and invited students to indicate again their most dominant achievement goal. Cross-tabulation of students' responses indicated that students responded quite consistently, Cramer's $V(428)=0.780, p<.01$. Specifically, $98.5 \%, 80.0 \%$, and $81.2 \%$ of the participants who initially selected, respectively, MAp, PAp, and PAv goals, indicated the same achievement goal as the most dominant one in the second round. 


\section{Underlying reasons of achievement goals}

Using the same set of items that we used in Study 1, we asked to what extent students pursued their dominant achievement goal for autonomous and controlling reasons.

\section{Motivated learning strategies}

As in Study 1, we used the same subscales from the MSLQ (Pintrich et al., 1993) to assess students' critical thinking, metacognitive self-regulation, and effort regulation.

\section{Cheating}

The Anderman, Griesinger, and Westerfield's (1998) scale was employed to assess students self-reported cheating behaviours (five items; e.g., 'I use cheat sheets when I take exams') and cheating beliefs (three items; e.g., 'It is ok to cheat during exams').

\section{Results}

\section{Preliminary analysis}

Contrary to what we had hoped, most students favoured MAp goal ( $n=411 ; 93.4 \%)$ over PAp $(n=10 ; 2.3 \%)$ and PAv goals $(n=16 ; 3.6 \%)$, while three students $(0.7 \%)$ failed to respond to the most dominant goal question. Nevertheless, this finding may come as no surprise given prior research (e.g., Lemos, 1996; Van Yperen, 2006) and theorizing (e.g., Brophy, 2005) claiming the pervasive role of mastery goals in educational contexts. Consequently, we retained only those participants who consistently reported a dominant MAp goal ( $N=400 ; 90.8 \%$ females), and we examined whether the variability in autonomous and controlling reasons within this group could be predicted by distal motives.

Descriptive statistics, bivariate correlations, and Cronbach alphas of the measured variables appear in Table 2. Confirmatory Factor Analysis of each scale showed acceptable fit, and the results are available upon request. A MANOVA test showed gender effects, Wilk's $\Lambda=.950, F(8,386)=2.54, p<.05$, multivariate $\eta^{2}=.05$. Follow-up ANOVA after Bonferroni's correction indicated significant gender differences in effort regulation $F(1,393)=9.23, p=.003, \eta^{2}=.02$ with males $(M=3.12$, $S D=0.82)$ reporting lower levels of effort regulation than females $(M=3.53$, $S D=0.73)$. Therefore, gender was included as a covariate in the main analyses.

\section{Main analyses}

Similar to Study 1, we performed path analysis with EQS 6.1 software to test the mediating role of autonomous and controlling reasons underlying the pursuit of the dominant MAp goal between the distal achievement motives and study-related outcomes. Taking into account the modifications we implemented in Study 1 (i.e., a direct path between need for achievement and two of the three outcomes) and similar to previous studies (e.g., Diseth \& Kobbeltvedt, 2010), we also included direct paths between need for achievement and the three learning strategies and we retained only the significant ones. Furthermore, the model fit was improved by allowing direct paths from fear of failure to critical thinking and cheating.

The obtained model (see Figure 3) showed acceptable fit (S-B $\chi^{2}$ [22, $N=396]=38.14, \quad p<.01, \quad$ CFI $=.966, \quad$ SRMR $=.052, \quad$ RMSEA $=.043 \quad[90 \% \quad$ CI: 


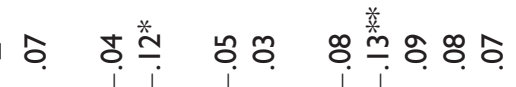

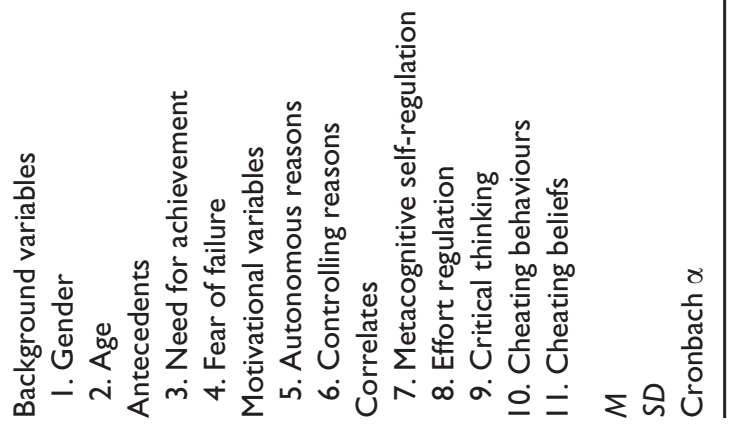




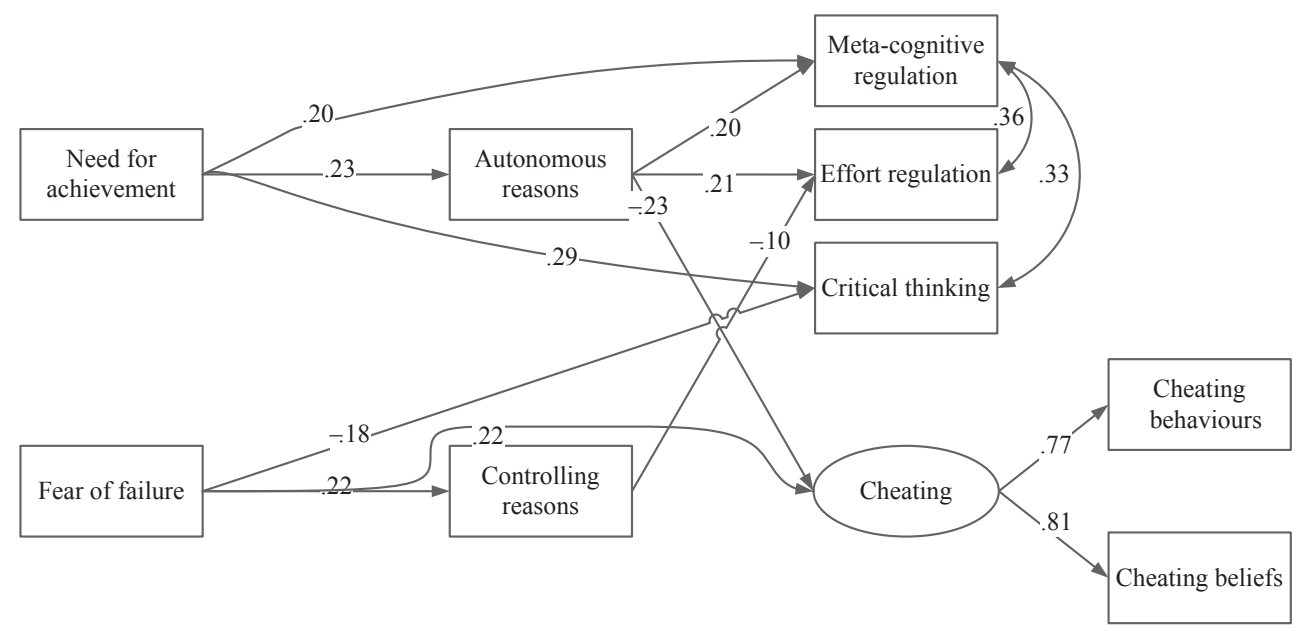

Figure 3. The Tested Model of Study 2 controlling for gender differences (not shown for sake of clarity). Also, not shown for sake of clarity are the correlations between autonomous and controlling reasons $(\beta=.20)$; cheating and metacognitive regulation $(\beta=-.3 \mathrm{I})$, effort regulation $(\beta=-.49)$ as well as critical thinking $(\beta=-.14)$. All paths are standardized and significant at the .05 level.

.018-.066]). Similar to Study 1, need for achievement was positively related to autonomous reasons and unrelated to controlling reasons underlying MAp dominant goal. In turn, autonomous reasons were positively associated to metacognitive selfregulation and effort regulation, but not with critical thinking. Additionally, a direct positive relation was observed between need for achievement and critical thinking and metacognitive self-regulation. In contrast, fear of failure was unrelated with autonomous reasons and positively correlated with controlling reasons for pursuing dominant MAp goals which in turn were associated negatively to effort regulation. Finally, a direct positive path was found between fear of failure and cheating, whereas a direct negative path linked fear of failure to critical thinking. A test of indirect effects showed that need for achievement was indirectly positively associated with effort regulation $(\beta=.05, z=3.08$, $p<.01)$ and metacognitive self-regulation $(\beta=.05, z=3.15, p<.01)$ and negatively to cheating $(\beta=-.06, z=-3.29, p<.01)$ by means of underlying autonomous reasons. In contrast, fear of failure was indirectly negatively associated, although marginally, with effort regulation $(\beta=-.02, z=-1.96, p=.05)$ by means of underlying controlling reasons.

\section{Brief discussion}

Study 2 was intended to examine whether need for achievement and fear of failure would predict variability in the reasons underlying every chosen dominant achievement goal. However, the vast majority of the participants chose MAp goal as their dominant achievement goal, which led us to examine the role of reasons in this group only. Autonomous reasons for MAp goals were predicted by the need for achievement and related positively to effective learning strategies and negatively to cheating. Controlling reasons for MAp goals were predicted by fear of failure and relate negatively to effort regulation. As these findings suggest, when students adopt MAp goals for controlling 
reasons, they are characterized by fear of failure and poor educational outcomes. Considering the reasons underlying the pursuit of achievement goals adds explanatory value to the hierarchical model of achievement motivation.

\section{GENERAL DISCUSSION}

Defining achievement goals (Elliot \& Thrash, 2001) as pure aims provides the conceptual basis for a more intensive study of the reasons underlying the pursuit of achievement goals. Grounded in SDT (Deci \& Ryan, 2000), previous work has shown that the autonomous or controlling reasons underlying learners' (Gaudreau, 2012; Vansteenkiste, Smeets, et al., 2010), soccer players' (Vansteenkiste, Mouratidis, et al., 2010) and workers' (Gillet et al., 2012) achievement goals matter in predicting people's functioning, in addition to the specific types of achievement goals they pursue. What has not been explored yet in past work is whether the goals as such and the reasons for pursuing these goals are rooted in the same motivational dispositions. The aim of the present study was to test within an integrated model if achievement goals, defined as pure aims, and their underlying autonomous and controlling reasons mediate the relation of need for achievement and fear of failure to learning outcomes. Two particular features of the tested enriched hierarchical model deserve being mentioned.

Aligned with the hierarchical model of achievement motivation (Elliot, 1999), in Study 1, need for achievement related positively to MAp and PAp goals and fear of failure related positively to both PAv and PAp goals. Building on the hierarchical model, we found need for achievement and fear of failure to predict the reasons underlying learners' achievement goals. Specifically, need for achievement related to the pursuit of an achievement goal for autonomous reasons and fear of failure seems to orient people to pursue achievement goals for controlling reasons. These findings were obtained in both Study 1, for which an aggregated measure of autonomous and controlling reasons was created, and Study 2, which zoomed on dominant MAp goals. Interestingly, need for achievement and fear of failure can predict variability in learners' reasons for pursuing mastery goals. Overall then, the distal motives of need for achievement and fear of failure do not only orient individuals to particular types of achievement goals, but also instigate certain reasons for pursuing these goals. Said differently, achievement motives also manifest through the type of reasons one has for pursuing achievement goals. This constitutes a novel aspect introduced by the proposed enriched hierarchical model of achievement motivation.

A second novel feature of the enriched hierarchical model is that the consideration of the reasons underlying achievement goals, in addition to the achievement goals themselves, helps to account for the relation between achievement motive dispositions and outcomes. ${ }^{4}$ In particular, on the one hand, only MAp goals (and not PAp and PAv goals) related to effective learning strategies once the autonomous and controlling reasons underlying goal pursuit were taken into account. On the other hand, autonomous

\footnotetext{
${ }^{4}$ To check whether the autonomous and controlling reasons, irrespectively of the achievement goal to which they are tied, are predicted by need for achievement and fear of failure, respectively, and predict, respectively, positive and negative outcomes, we disregarded the problem of multicollinearity in Study I and tested a model with the three achievement goals as well as the three autonomous and the three controlling reasons as distinct mediators. We found autonomous and controlling reasons, irrespectively of the achievement goal to which they are tied, to relate to need for achievement and fear of failure, respectively. Regarding the relation with the outcomes, we found only MAp goals and autonomous reasons for pursuing MAp and PAp goals to mediate the relation between need for achievement and effective learning outcomes, suggesting a qualitative distinction among autonomous reasons underlying approach goals relative to autonomous reasons underlying PAv goals.
} 
reasons related positively to effective learning strategies (Study 1 and 2) and negatively to cheating (Study 2), whereas controlling reasons related negatively to effort regulation (yet only in Study 2) and were unrelated to cheating. This pattern of associations provides a more refined picture about achievement motivation as it shows that not only MAp goals but also autonomous reasons for adopting achievement goals are positive motivational constructs.

Three additional findings deserve discussion. First, some differences emerged in the models of the two studies. Specifically, different from Study 1, controlling reasons for MAp goals in Study 2 were negatively related to effort regulation, whereas autonomous reasons for MAp goals were not related to critical thinking. These differences could be attributed to the fact that in Study 1, we did not investigate separately the relation of autonomous and controlling reasons for MAp goals to the outcomes, but we used composite scores for autonomous and controlling reasons underlying different achievement goals.

Second, the distal achievement motives in our studies appeared as strong dispositions that manifested themselves not only indirectly through the achievement goals and their underlying reasons to learning outcomes but directly as well. For example, in Study 2, fear of failure did positively predict cheating and negatively critical thinking while need for achievement did positively predict critical thinking. The proposed enriched hierarchical model of achievement motivation contributes to a better understanding of how the achievement outcomes occur. Our findings suggest that distal motives as well as proximal reasons, next to achievement goals, account for learning outcomes.

Third, the strong positive correlations among the autonomous and among the controlling reasons for pursuing different types of achievement goals could imply that students regulate every achievement goal they pursue in the same way, depending on the strength of their achievement motive dispositions (see also Gaudreau, 2012). Although we tried to address this issue in Study 2, more studies are needed to examine whether the same reasons govern one's achievement striving, irrespective of the goals one endorses. Future research need to devise other ways to empirically separate the reasons underlying the pursuit of different achievement goals. For instance, diary studies that will assess intrapersonal variation in goal pursuit and the reasons underlying this pursuit may more effectively address this issue - see Vansteenkiste, Mouratidis, Van Riet, and Lens (2014) for an example in the sport domain.

\section{Limitations}

The cross-sectional design of our research prevents us from claiming cause-effect phenomena. Experimental studies or long-term longitudinal studies can better test the causal relationships among distal motives, achievement goals, their proximal underlying reasons, and outcomes. Another limitation concerns the generalizability of the findings in other cultures, contexts, and population samples. Future studies are needed to test the enriched hierarchical model of achievement motivation, controlling for social desirability, in different settings like sport and work.

\section{References}

Anderman, E. M., Griesinger, T., \& Westerfield, G. (1998). Motivation and cheating during early adolescence. Journal of Educational Psychology, 90, 84-93. doi:10.1037/0022-0663.90.1.84 
Atkinson, J. W. (1957). Motivational determinants of risk-taking behavior. Psychology Review, 64, 359-372.

Atkinson, J. W., \& Feather, N. T. (1966). A theory of achievement motivation. New York, NY: Wiley. Benita, M., Roth, G., \& Deci, E. L. (2014). When are mastery goals more adaptive? It depends on experiences of autonomy support and autonomy. Journal of Educational Psychology, 106, 258-267. doi:10.1037/a0034007

Brophy, J. (2005). Goal theorists should move on from performance goals. Educational Psychologist, 40, 167-176. doi:10.1207/s15326985ep4003_3

Ciani, K. D., \& Sheldon, K. M. (2010). Evaluating the mastery-avoidance construct: A study of elite college baseball players. Psychology of Sport and Exercise, 11, 127-132. doi:10.1016/j. psychsport.2009.04.005

Darnon, C., Dompnier, B., Delmas, F., Pulfrey, C., \& Butera, F. (2009). Achievement goal promotion at university: Social desirability and social utility of mastery and performance goals. Journal of Personality and Social Psychology, 96, 119-134. doi:10.1037/a0012824

Deci, E. L., \& Ryan, R. M. (2000). The "What" and "Why" of goal pursuits: Human needs and the self determination of behavior. Psychological Inquiry, 11, 227-268. doi:10.1207/ S15327965pli1104_01

Diseth, $\AA .$, \& Kobbeltvedt, T. (2010). A mediation analysis of achievement motives, goals, learning strategies, and academic achievement. British Journal of Educational Psychology, 80, 671687. doi:10.1348/000709910X492432

Elliot, A. J. (1999). Approach and avoidance motivation and achievement goals. Educational Psychologist, 34, 169-189. doi:10.1207/s15326985ep3403_3

Elliot, A. J. (2005). A conceptual history of the achievement goal construct. In A. Elliot \& C. Dweck (Eds.), Handbook of competence and motivation (pp. 52-72). New York, NY: Guilford.

Elliot, A. G. (2006). The hierarchical model of approach-avoidance motivation. Motivation and Emotion, 30, 111-116. doi:10.1007/s11031-006-9028-7

Elliot, A. J., \& Church, M. A. (1997). A hierarchical model of approach and avoidance achievement motivation. Journal of Personality and Social Psychology, 72, 218-232. doi: 10.1037/0022-3514.72.1.218

Elliot, A. J., \& Murayama, K. (2008). On the measurement of achievement goals: Critique, illustration, and application. Journal of Educational Psychology, 100, 613-628. doi:10.1037/0022-0663. 100.3.613

Elliot, A. J., \& Thrash, T. M. (2001). Achievement goals and the hierarchical model of achievement motivation. Educational Psychology Review, 13, 139-156. doi:10.1037/0022-3514.82.5.804

Gaudreau, P. (2012). Goal self-concordance moderates the relationship between achievement goals and indicators of academic adjustment. Learning and Individual Differences, 22, 827-832. doi:10.1016/j.lindif.2012.06.006

Gillet, N., Lafreniere, M.-A. K., Vallerand, R. J., Huart, I., \& Fouquereau, E. (2012). The effects of autonomous and controlled regulation of performance-approach goals on well-being: A process model. British Journal of Social Psychology, 51, 1-21. doi:10.1111/bjso.12018

Hambleton, R. K. (1994). Guidelines for adapting educational and psychological tests: A progress report. European Journal of Psychological Assessment, 10, 229-240.

Hulleman, C. S., Schrager, S. M., Bodmann, S. M., \& Harackiewicz, J. M. (2010). A meta-analytic review of achievement goal measures: Different labels for the same constructs or different constructs with similar labels? Psychological Bulletin, 136, 422-449. doi:10.1037/a0018947

Husman, J., \& Lens, W. (1999). The role of the future in student motivation. Educational Psychologist, 34, 113-125. doi:10.1207/s15326985ep3402_4

Lang, J. W. B., \& Fries, S. (2006). A Revised ten item version of Achievement Motives scale. European Journal of Psychological Assessment, 22, 216-224. doi:10.1027/1015-5759.22.3.216

Lemos, M. S. (1996). Students' and teachers' goals in the classroom. Learning and Instruction, 6 , 151-171.

Lewin, K. (1938). The conceptual representation and the measurement of psychological forces. Durham, NC: Duke University Press. 
McGregor, H. A., \& Elliot, A. J. (2005). The shame of failure: Examining the link between fear of failure and shame. Personality and Social Psychology Bulletin, 31, 218-231. doi:10.1177/ 0146167204271420

Michou, A., Mouratidis, A., Lens, W., \& Vansteenkiste, M. (2013). Personal and contextual antecedents of achievement goals: Their direct and indirect relations to students' learning strategies. Learning and Individual Differences, 23, 187-194. doi:10.1016/j.lindif.2012.09. 005

Pintrich, P. R., Smith, D., Garcia, T., \& McKeachie, W. J. (1993). Reliability and predictive validity of the Motivated Strategies for Learning Questionnaire (MSLQ). Educational and Psychological Measurement, 53, 801-813. doi:10.1177/0013164493053003024

Sheldon, K. M., \& Cooper, M. L. (2008). Goal striving within agentic and communal roles: Separate but functionally similar pathways to enhanced well-being. Journal of Personality, 76, 415-447. doi:10.1111/j.1467-6494.2008.00491.x

Urdan, T., \& Mestas, M. (2006). The goals behind performance goals. Journal of Educational Psychology, 98, 354-365. doi:10.1037/0022-0663.98.2.354

Van Yperen, N. W. (2006). A novel approach to assessing achievement goals in the context of the 2 X 2 framework: Identifying distinct profiles of individuals with different dominant achievement goals. Personality and Social Psychology Bulletin, 32, 1432-1445. doi:10.1177/0146167206292093

Vansteenkiste, M., Lens, W., Elliot, A. J., Soenens, B., \& Mouratidis, A. (2014). Moving the achievement goal approach one step forward: Toward a systematic examination of the autonomous and controlled reasons underlying achievement goals. Educational Psychologist, 49, 153-174. doi:10.1080/00461520.2014.928598

Vansteenkiste, M., Mouratidis, A., \& Lens, W. (2010). Detaching reasons from aims: Fair play and well-being in soccer as a function of pursuing performance-approach goals for autonomous or controlling reason. Journal of Sport E Exercise Psychology, 32, 217-242.

Vansteenkiste, M., Mouratidis, A., Van Riet, T., \& Lens, W. (2014). Examining correlates of game-to-game variation in volleyball players' achievement goal pursuit and underlying autonomous and controlling reasons. Journal of Sport \& Exercise Psychology, 36, 131-145.

Vansteenkiste, M., Smeets, S., Lens, W., Soenens, B., Matos, L., \& Deci, E. L. (2010). Autonomous and controlled regulation of performance-approach goals: Their relations to perfectionism and educational outcomes. Motivation and Emotion, 34, 333-353. doi:10.1007/s11031-010-9188-3 